Akihiko Morimoto

Nagoya Math. J.

Vol. 40 (1970), 13-31

\title{
LIFTINGS OF SOME TYPES OF TENSOR FIELDS AND GONNEGTIONS TO TANGENT BUNDLES OF $\mathrm{p}^{r}$-VELOGITIES
}

\author{
AKIHIKO MORIMOTO
}

\section{Introduction.}

In the previous paper [6], we studied the liftings of tensor fields to tangent bundles of higher order. The purpose of the present paper is to generalize the results of [6] to the tangent bundles $\stackrel{r, p}{T} M$ of $p^{r}$-velocities in a manifold $M-$ notions due to C. Ehresmann [1] (see also [2]). In §1, we explain the $p^{r}$-velocities in a manifold and define the ( $\lambda$ )-lifting of differentiable functions for any multi-index $\lambda=\left(\lambda_{1}, \lambda_{2}, \cdots, \lambda_{p}\right)$ of non-negative integers $\lambda_{i}$ satisfying $\sum \lambda_{i} \leq r$. In $\S 2$, we construct $\langle\lambda\rangle$-lifts of any vector fields and

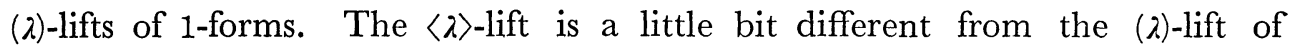
vector fields in [6].

In $\S 3$, we construct $(\lambda)$-lifting of $(0, q)$-tensor fields and then ( $\lambda$ )-lifting of $(1, q)$-tensor fields to $\stackrel{r, p}{T} M$ for $q \geq 1$. Unfortunately, the author could not construct a natural lifting of $(s, q)$-tensor fields to $\stackrel{r, p}{T} M$ for $s \geq 2$.

Nevertheless, our ( $\lambda$ )-liftings of $(s, q)$-tensor fields for $s=0$ or 1 are quite sufficient for the geometric applications, because the important tensor fields with which we encounter so far in differential geometry seem to be, fortunately, only of type $(s, q)$ with $s=0$ or 1 .

As an application, we shall consider in $\$ 4$, the prolongations of almost complex structures and prove that if $M$ is a (homogeneous) complex manifold, then $\stackrel{r, p}{T} M$ is also a (homogeneous) complex manifold.

In $\S 5$, we consider the liftings of affine connections to $\stackrel{r, p}{T} M$ and prove that if $M$ is locally affine symmetric then $\stackrel{r, p}{T} M$ is also locally affine symmetric with respect to the lifted affine connection.

In $\S 6$, we shall give a proof for the fact that if $M$ is an affine symmetric space then $\stackrel{r, p}{T} M$ is also an affine symmetric space.

Received June 17, 1969. 
In this paper, all manifolds and mappings (functions) are assumed to be differentiable of class $C^{\infty}$, unless otherwise stated.

We shall fix two positive integers $r$ and $p$ throughout the paper.

\section{§1. Tangent bundles of $p^{r}$-velocities.}

Consider the algebra $C^{\infty}\left(R^{p}\right)$ of all $C^{\infty}$-functions on the $p$-dimensional euclidean space $R^{p}$ with natural coordinates $\left(t_{1}, t_{2}, \cdots, t_{p}\right)$. For any $p$-tuple $\nu=\left(\nu_{1}, \nu_{2}, \cdots, \nu_{p}\right)$ of non-negative integers $\nu_{j}$ we denote as usual by $(\partial / \partial t)^{\nu}$ the following partial differentiation

$$
\left(\frac{\partial}{\partial t}\right)^{\nu} f=\frac{\partial^{\nu_{1}+\cdots+\nu_{p}} f}{\partial t_{1}^{\nu_{1}} \cdots \cdot \partial t_{p}^{\nu_{p}}}
$$

for $f \in C^{\infty}\left(R^{p}\right)$. We define $|\nu|$ and $\nu$ ! as follows:

$$
|\nu|=\nu_{1}+\cdots+\nu_{p}, \nu !=\nu_{1} ! \nu_{2} ! \cdots \nu_{p} ! \text {. }
$$

We denote by $N(p, r)$ the set of all $p$-tuples $\nu=\left(\nu_{1}, \cdots, \nu_{p}\right)$ of nonnegative integers $\nu_{i}$ such that $|\nu| \leq r$. The set $N(p, r)$ is a subset of the additive group $Z^{p}$ of all $p$-tuples of integers.

Take two elements $f, g \in C^{\infty}\left(R^{p}\right)$. We say $f$ is $r$-equivalent to $g$ if $\langle\partial / \partial t)^{\nu} f=(\partial / \partial t)^{\nu} g$ at $t=\left(t_{1}, \cdots, t_{p}\right)=0$ for every $\nu \in N(p, r)$ and denote it by $f \underset{r}{\sim}$. Clearly $\underset{r}{\sim}$ is an equivalence relation in $C^{\infty}\left(R^{p}\right)$.

Now, let $M$ be an $n$-dimensional manifold. Consider the set $S_{p}(M)$ of all maps $\varphi: R^{p} \rightarrow M$. Take two elements $\varphi, \phi \in S_{p}(M)$. We say that $\varphi$ is $r$-equivalent to $\phi$ if $f \circ \varphi \underset{r}{\sim} f \circ \phi$ for every $f \in C^{\infty}(M)$ and denote it by $\varphi \underset{r}{\sim} \phi$. The relation $\sim$ is also an equivalence relation in $S_{p}(M)$. We denote by ${ }^{r, p} M$ the set of ${ }^{r}$ all equivalence classes in $S_{p}(M)$ with respect to the equivalence relation $\underset{r}{\sim}$. We denote by $[\varphi]_{r}$ the equivalence class containing $\varphi \in S_{p}(M)$, and we shall call it a $p^{r}$-velocity in $M$ at $\varphi(0)$. To introduce the manifold structure in $\stackrel{r, p}{T} M$, we define local coordinate system on $\stackrel{r, p}{T} M$ as follows: Take a coordinate neighborhood $U$ in $M$ with coordinate system $\left\{x_{1}, x_{2}, \cdots, x_{n}\right\}$. Define the coordinate functions $\left\{x_{i}^{(\nu)} \mid i=1, \cdots, n ; \nu \in N(p, r)\right\}$ on $\stackrel{r}{T} p$

$$
x_{i}^{(\nu)}\left([\varphi]_{r}\right)=\frac{1}{\nu !}\left[\left(\frac{\partial}{\partial t}\right)^{\nu}\left(x_{i} \circ \varphi\right)\right]_{t=0}
$$

for $[\varphi]_{r} \in \stackrel{r, p}{T} U$ (cf. (1.1)). It is straightforward to see that $\stackrel{r, p}{T} M$ becomes a manifold by the above coordinate systems $\left\{x_{i}^{(\nu)}\right\}$. The projection $\stackrel{r, p}{\pi}$ defined 
by $\stackrel{r, q}{\pi}\left([\varphi]_{r}\right)=\varphi(0)$ for $\varphi \in S_{p}(M)$ is clearly a differentiable map of $\stackrel{r, p}{T} M$ onto $M$.

Definition 1.1. The differentiable manifold $\stackrel{r, p}{T} M$ with projection $\stackrel{r, p}{\pi}$ will be called the tangent bundle of $p^{r}$-velocities in $M$.

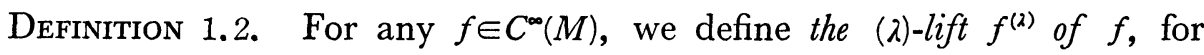
every $\lambda \in N(p, r)$, as follows:

$$
f^{(\lambda)}\left([\varphi]_{r}\right)=\frac{1}{\lambda !}\left[\left(\frac{\partial}{\partial t}\right)^{2}(f \circ \varphi)\right]_{t=0}
$$

for $[\varphi]_{r} \stackrel{r, p}{\in T} M$. Clearly, $f^{(2)}$ is a well-defined differentiable function on $\stackrel{r, p}{T} M$. We note also that $\left(x_{i}\right)^{(\nu)}=x_{i}^{(\nu)}$ holds on $\stackrel{r, p}{T} U$ for the above coordinate system $\left\{x_{1}, \cdots, x_{n}\right\}$.

For the sake of convenience we define $f^{(\lambda)}=0$ for any $\lambda \in Z^{p}$ such that $\lambda \notin N(p, r)$.

Lemma 1.3. The ( $\lambda$-lifting $f \rightarrow f^{(2)}$ is a linear map of $C^{\infty}(M)$ into $C^{\infty}(\stackrel{r, p}{T} M)$ and satisfies the following equality

$$
(f \cdot g)^{(\lambda)}=\sum_{\mu \in Z^{p}} f^{(\mu)} \cdot g^{(\lambda-\mu)}
$$

for every $f, g \in C^{\infty}(M)$ and $\lambda \in N(p, r)$.

Proof. Straightforward verification similar to the one of Lemma 1.2 [6].

\section{§2. Liftings of vector fields and 1-forms.}

Let $\mathscr{T}(M)=\sum \mathscr{T}_{q}^{s}(M)$ be, as in [6], the tensor algebra of all tensor fields on $M$.

Lemma 2.1. For any $X \in \mathscr{T}_{0}^{1}(M)$ and any $\lambda \in N(p, r)$ there exists one and only one $\left.X^{<\lambda>} \in \mathscr{T}_{0}^{1} \stackrel{r, p}{(T M} M\right)$ satisfying the following equality

$$
X^{<\lambda>} f^{(\mu)}=(X f)^{(\mu-\lambda)}
$$

for every $f \in C^{\infty}(M)$ and $\mu \in N(p, r)$.

Proof. Take a coordinate neighborhood $U$ in $M$ with coordinate system $\left\{x_{1}, \cdots, x_{n}\right\}$ and let $X=\sum a_{i} \cdot \partial / \partial x_{i}\left(a_{i} \in C^{\infty}(U)\right)$ be the local expression of $X$ in $U$. Consider the vector field $\tilde{X}=\tilde{X}_{U}$ on $(\pi, p)$ defined by 


$$
\tilde{X}=\sum_{\mu \in N(p, r)} \sum_{j=1}^{n} a_{j}^{(\mu-\lambda)} \frac{\partial}{\partial x_{j}^{(\mu)}}
$$

We see that $\tilde{X}\left(x_{j}^{(\mu)}\right)=a_{j}^{(\mu-\lambda)}=\left(X x_{j}\right)^{(\mu-\alpha)}$ for $j=1,2, \cdots, n ; \mu \in N(p, r)$. Now, making use of same arguments as in the proof of Lemma 1.4 [6], we can prove that $\tilde{X}\left(f^{(\mu)}\right)=(X f)^{(\mu-\lambda)}$ for every $f \in C^{\infty}(U)$ and $\mu \in N(p, r)$. We can also prove that if $U^{\prime}$ is a coordinate neighborhood in $M$ such that $U \cap U^{\prime}=U^{\prime \prime} \neq \phi$, then $\tilde{X}_{U}\left|U^{\prime \prime}=\tilde{X}_{U}\right| U^{\prime \prime}$ holds. Thus we obtain a vector field $X^{<\lambda>}$ on $\stackrel{r, p}{T} M$ such that $X^{<\lambda>} \mid\left({ }^{r, p} \pi\right)^{-1}(U)=\tilde{X}_{U}$ for every coordinate neighborhood $U$ in $M$. This vecotr field $X^{<\lambda>}$ clearly satisfies the condition (2.1) for every $f \in C^{\infty}(M)$ and $\mu \in N(p, r)$. The uniqueness of $X^{<\lambda>}$ is also easily verified. Q.E.D.

Corollary 2.2. Let $\left\{x_{1}, \cdots, x_{n}\right\}$ be a local coordinate system on a neighborhood $U$ in $M$. Then, we have

$$
\left(\frac{\partial}{\partial x_{i}}\right)^{\langle\lambda\rangle}=\frac{\partial}{\partial x_{i}^{(\lambda)}}
$$

for every $i=1, \cdots, n$ and $\lambda \in N(p, r)$.

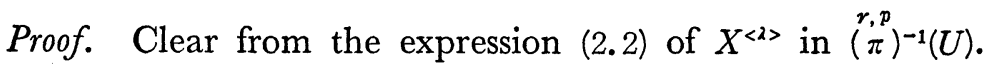

Corollary 2.3. Notations being as in Corollary 2.2, we have

$$
\left(\frac{\partial f}{\partial x_{i}}\right)^{(\lambda-\mu)}=\frac{\partial f^{(\lambda)}}{\partial x_{i}^{(\mu)}}
$$

for every $i=1, \cdots, n$ and $\lambda, \mu \in N(p, r)$.

Proof. By Corollary 2.2, we have

$$
\frac{\partial f^{(\lambda)}}{\partial x_{i}^{(\mu)}}=\left(\frac{\partial}{\partial x_{i}}\right)^{\langle\mu\rangle} f^{(\lambda)}=\left(\frac{\partial f}{\partial x_{i}}\right)^{(\lambda-\mu)} .
$$

Definition 2.4. The vector field $X^{<\lambda>}$ in Lemma 2.1 will be called the $\langle\lambda\rangle$-lift of $X$ to $\stackrel{r, p}{T} M$ for $\lambda \in N(p, r)$. For the sake of convenience, we define $X^{<\lambda\rangle}=0$ for every $\lambda \in Z^{p}$ such that $\lambda \notin N(p, r)$. The $\langle\lambda\rangle$-lifting $X \rightarrow X^{<\lambda\rangle}$ is a linear map of $\mathscr{T}_{0}^{1}(M)$ into $\left.\mathscr{T}_{0}^{1} \stackrel{r \cdot p}{(T} M\right)$ for every $\lambda \in Z^{p}$.

Lemma 2.5. For $X, Y \in \mathscr{T}_{0}^{1}(M)$, we have

$$
\left[X^{<\lambda\rangle}, Y^{<\mu>}\right]=[X, Y]^{<\lambda+\mu>}
$$

for every $\lambda, \mu \in N(p, r)$. 
Proof. Assume $\lambda+\mu \in N(p, r)$. Then, for any $g \in C^{\infty}(M)$ and $\nu \in N(p, r)$ we have

$$
\begin{aligned}
& {\left[X^{\langle\lambda\rangle}, Y^{\langle\mu\rangle}\right]\left(g^{(\nu)}\right)=X^{\langle\lambda\rangle} Y^{\langle\mu\rangle} g^{(\nu)}-Y^{\langle\mu\rangle} X^{\langle\lambda\rangle} g^{(\nu)}} \\
& =X^{\langle\lambda\rangle}(Y g)^{(\nu-\mu)}-Y^{\langle\mu>}(X g)^{(\nu-\mu)} \\
& =X^{\langle\lambda\rangle}(Y g)^{(\nu-\mu)}-Y^{\langle\mu>}(X g)^{(\nu-\lambda)} \\
& =(X Y g-Y X g)^{(\nu-\lambda-\mu)}=([X, Y] g)^{(\nu-\lambda-\mu)} \\
& =[X, Y]^{<\lambda+\mu>} g^{(\nu)} .
\end{aligned}
$$

Since $g \in C^{\infty}(M)$ and $\nu \in N(p, r)$ are arbitrary we get (2.5) if $\lambda+\mu \in N(p, r)$.

Assume $\lambda+\mu \notin N(p, r)$, then by our convention, we have $[X, Y]^{<\lambda+\mu>}=0$. On the other hand, for any $g \in C^{\infty}(M)$ and $\nu \in N(p, r)$ we have, by the same calculation as above,

$\left[X^{<\lambda\rangle}, Y^{<\mu>}\right] g^{(\nu)}=(X Y g)^{(\nu-\mu-\lambda)}-(Y X g)^{(\nu-\lambda-\mu)}=0$, since $\nu-\mu-\lambda \notin N(p, r)$. Thus (2.5) is verified in any case.

Q.E.D.

Lemma 2.6. For $X \in \mathscr{T}_{0}^{1}(M)$ and $f \in \mathscr{T}_{0}^{0}(M)$, we have

$$
(f \cdot X)^{<\lambda>}=\sum_{\nu \in N(p, r)} f^{(\nu)} \cdot X^{<\lambda+\nu>}
$$

for every $\lambda \in N(p, r)$.

Proof. For any $g \in \mathscr{T}_{0}^{0}(M)$ and $\mu \in N(p, r)$, we have

$$
\begin{aligned}
& (f \cdot X)^{\langle\lambda\rangle} g^{(\mu)}=(f X \cdot g)^{(\mu-\lambda)}=(f \cdot X g)^{(\mu-\lambda)} \\
& =\sum_{\nu \in Z^{p}} f^{(\nu)} \cdot(X g)^{(\mu-\lambda-\nu)}=\sum f^{(\nu)} \cdot X^{\langle\lambda+\nu\rangle} g^{(\mu)} \\
& =\left(\sum f^{(\nu)} X^{<\lambda+\nu>}\right) g^{(\mu)} .
\end{aligned}
$$

Since $g$ and $\mu$ are arbitrary, we get (2.6) for every $\lambda \in N(p, r)$. Q.E.D.

Remark 2.7. By our convention (cf. Def. 1.2) we can write (2.6) as follows :

$$
(f \cdot X)^{<\lambda>}=\sum_{\nu \in Z^{p}} f^{(\nu)} X^{<\lambda+\nu>}
$$

LEMMA 2.8. Let $f_{i}, g_{i} \in C^{\infty}(M)(i=1, \cdots, k)$ be such that $\sum g_{i} d f_{i}=0$ on M. Then the following equality

$$
\sum_{i=1}^{k} \sum_{\mu \in Z^{p}} g_{i}^{(\mu)} d f_{i}^{(2-\mu)}=0
$$


holds on $\stackrel{r, p}{T} M$ for every $\lambda \in N(p, r)$.

Proof. Similar to the proof of Lemma 2.1 [6].

LEMmA 2.9. There is one and only one lifting $L_{\lambda}: \mathscr{T}_{1}^{0}(M) \rightarrow \mathscr{T}_{1}^{0}(\stackrel{p}{T} M)$ for every $\lambda \in N(p, r)$ satisfying the following condition:

$$
L_{\lambda}(f \cdot d g)=\sum_{\lambda \in Z^{p}} f^{(\mu)} d g^{(\lambda-\mu)}
$$

for every $f, g \in \mathscr{T}_{0}^{0}(M)$.

Proof. Similar to the proof of Lemma 2.2 [6].

Lemma 2.10. For $f \in \mathscr{T}_{0}^{0}(M)$ and $\theta \in \mathscr{T}_{1}^{0}(M)$, we have

$$
(f \cdot \theta)^{(\lambda)}=\sum_{\mu \in Z^{p}} f^{(\mu)} \cdot \theta^{(\lambda-\mu)}
$$

for every $\lambda \in N(p, r)$.

Proof. Similar to the proof of Corollary 2.4 [6].

Lemma 2.11. For $\theta \in \mathscr{T}_{1}^{0}(M)$ and $X \in \mathscr{T}_{0}^{1}(M)$, we have

$$
\theta^{(\lambda)}\left(X^{<\mu>}\right)=(\theta(X))^{(\lambda-\mu)}
$$

for every $\lambda, \mu \in N(p, r)$.

Proof. Let $\theta=\sum f_{i} d x_{i}$ be the local expression of $\theta$. Making use of Lemma 2.1, we calculate as follows:

$$
\begin{aligned}
& \theta^{(\lambda)}\left(X^{\langle\mu \nu}\right)=\left(\sum f_{i} d x_{i}\right)^{(\lambda)}\left(X^{\langle\mu \nu}\right) \\
= & \sum_{i} \sum_{\nu \in Z^{p}} f_{i}^{(\nu)} d x_{i}^{(\lambda-\nu)}\left(X^{<\mu>}\right) \\
= & \sum_{i} \sum_{\nu} f_{i}^{(\nu)}\left(X^{<\mu>} x_{i}^{(\lambda-\nu)}\right)=\sum_{i} \sum_{\nu} f_{i}^{(\nu)}\left(X x_{i}\right)^{(\lambda-\nu-\mu)} \\
= & \sum_{i} \sum_{\nu} f_{i}^{(\nu)}\left(d x_{i}(X)\right)^{(\lambda-\nu-\mu)} \\
= & \sum\left(f_{i} \cdot d x_{i}(X)\right)^{(\lambda-\mu)}=(\theta(X))^{(\lambda-\mu)} .
\end{aligned}
$$

\section{§ 3. Lifting of $(1, q)$-tensor fields.}

Let $\mathscr{T}_{*}(M)$ be the subalgebra of $\mathscr{T}(M)$ consisting of all covariant tensor fields on $M$. We denote by $\underset{\mathscr{T}_{*}}{r \cdot p}$ the $m(r, p)$ times direct sum of $\stackrel{r, p}{\mathscr{\mathscr { S }}}(T M)$, where $m(r, p)$ denotes the number of elements in $N(p, r)$. i.e. 


$$
\stackrel{r, p}{\mathscr{T}}_{*}(M)=\sum_{q=0}^{\infty} \sum_{\lambda \in N(p, r)}\left(\mathscr{T}_{q}^{0}(\stackrel{r, p}{T} M)\right)_{\lambda}
$$

where $\left.\left(\mathscr{T}_{q}^{r, p}(T M)\right)_{\lambda}=\mathscr{T}_{q}^{0} \stackrel{r, p}{(T M} M\right)$ for all $\lambda \in N(p, r)$.

Take two elements $\theta=\left(\theta^{\lambda}\right)$ and $\eta=\left(\eta^{2}\right)$ in $\stackrel{r, p}{\mathscr{I}_{*}}(M)$. We define the multiplication $\theta \otimes \eta$ of $\theta$ and $\eta$ by the following:

$$
(\theta \otimes \eta)^{\lambda}=\sum_{\mu, \lambda-\mu \in N(p, r)} \theta^{\mu} \otimes \eta^{\lambda-\mu}
$$

for $\lambda \in N(p, r)$. We can readily see that $\stackrel{r, p}{\mathscr{\mathscr { T }}}(M)$ is an associative graded algebra over $\left.C^{\infty} \stackrel{r, p}{T} M\right)$ by this multiplication $\otimes$.

We have defined, in Lemma 2.9, the lifting $L_{\lambda}$ of $\mathscr{T}_{1}^{0}(M)$ into $\mathscr{T}_{1}^{r, p}(T M)$ for $\lambda \in N(p, r)$. Define $L: \mathscr{T}_{1}^{0}(M) \rightarrow \stackrel{r, p}{\mathscr{T}_{*}}(T M)$ by $L(\theta)=\left(L_{\lambda}(\theta)\right)_{\lambda \in N(p, r)}$ for $\theta \in T_{1}^{0}(M)$.

LEMma 3.1. There exists one and only one homomorphism $\tilde{L}: \mathscr{T}_{*}(M) \rightarrow \stackrel{r, p}{\mathscr{T}}(M)$ such that $\tilde{L} \mid \mathscr{T}_{1}^{0}(M)=L$.

Proof. Define $L^{q}:\left(\mathscr{T}_{1}^{0}(M)\right)^{q} \rightarrow \stackrel{r, p}{\mathscr{T}}_{*}(M)$ by

$$
L^{q}\left(\theta_{1}, \cdots, \theta_{q}\right)=L\left(\theta_{1}\right) \otimes \cdots \otimes L\left(\theta_{q}\right)
$$

for $\theta_{i} \in \mathscr{T}_{1}^{0}(M) i=1,2, \cdots, q$. Then, $L$ is a multilinear map satisfying the following condition:

$$
L^{q}\left(f_{1} \theta_{1}, \cdots, f_{q} \theta_{q}\right)=L\left(f_{1} \cdots f_{q}\right) \otimes L^{q}\left(\theta_{1}, \cdots, \theta_{q}\right)
$$

for $\theta_{i} \in \mathscr{T}_{1}^{0}(M)$ and $f_{i} \in \mathscr{T}_{0}^{0}(M) i=1, \cdots, q$, from which we conclude that there is a linear map $\tilde{L}^{q}$ of $\mathscr{\mathscr { T }}_{q}^{r, p}(M)$ into $\mathscr{T}_{*}(M)$ such that

$$
\tilde{L}^{q}\left(\theta_{1} \otimes \cdots \otimes \theta_{q}\right)=L\left(\theta_{1}\right) \otimes \cdots \otimes L\left(\theta_{q}\right)
$$

for $\theta_{i} \in \mathscr{T}_{1}^{0}(M), i=1, \cdots, q$. Thus $\tilde{L}^{q}(q \geqq 0)$ define a homomorphism $\tilde{L}: \stackrel{r, p}{\mathscr{T}}(M) \rightarrow \mathscr{T}_{*}(M)$ such that $\tilde{L}(\theta)=L(\theta)$ for $\theta \in \mathscr{T}_{1}^{0}(M)$.

Q.E.D.

Definition 3.2. For $K \in \mathscr{T}_{q}^{0}(M)$ we denote by $K^{(\lambda)}$ the $\lambda$-component of $\tilde{L}(K)$ for $\lambda \in N(p, r)$, i.e.

$$
\widetilde{L}(K)=\left(K^{(\lambda)}\right) \text {. }
$$

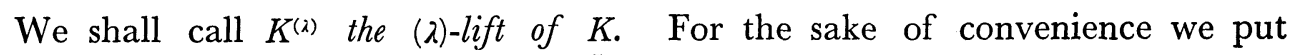
$K^{(\lambda)}=0$ for $\lambda \in Z^{p}$ such that $\lambda \notin N(p, r)$. 
Lemma 3.3. The notation $\alpha_{X}^{k}$ being as in Lemma 3.7 [6], for any $K \in \mathscr{T}_{q}^{0}(M)$ and $X \in \mathscr{T}_{0}^{1}(M)$, we have

$$
\alpha_{X}^{k}<\lambda>K^{(\mu)}=\left(\alpha_{X}^{k} K\right)^{(\mu-\lambda)}
$$

for $\lambda, \mu \in N(p, r)$.

Proof. Using Lemma 2.11, we can prove the lemma in the same way as the one of Lemma 3.7 [6].

Corollary 3.4. For $K \in \mathscr{T}_{q}^{0}(M)$ and $X_{i} \in \mathscr{T}_{0}^{1}(M) i=1, \cdots, q$, we have

$$
K^{(\lambda)}\left(X_{1}^{<\mu_{1}>}, \cdots, X_{q}^{<\mu_{q}>}\right)=\left(K\left(X_{1}, \cdots, X_{q}\right)\right)^{\left(\lambda-\Sigma \mu_{\sharp}\right)}
$$

for every $\lambda, \mu_{i} \in N(p, r), i=1, \cdots, q$.

Proof. We use Lemma 3.3 q-times.

Lemma 3.5. For any $K \in \mathscr{T}_{q}^{1}(M)$ and $\nu \in N(p, r)$, there is a unique $\widetilde{K}=K^{(\nu)} \in \mathscr{T}_{q}^{1} \stackrel{p, p}{(T)}$ such that

$$
\tilde{K}\left(X_{1}^{<\lambda_{1}>}, \cdots, X_{q}^{<\lambda_{q}>}\right)=\left(K\left(X_{1}, \cdots, X_{q}\right)\right)^{<\lambda+\nu>}
$$

for every $X_{i} \in \mathscr{T}_{0}^{1}(M)$ and $\lambda_{i} \in N(p, r)$, where $\lambda=\sum_{i} \lambda_{i}$.

Proof. Define $\left.\tilde{L}_{\nu}: \mathscr{T}_{0}^{1}(M) \times \mathscr{T}_{q}^{0}(M) \rightarrow \mathscr{T}_{q}^{1} \stackrel{r, p}{(T M} M\right)$ by the following

$$
\tilde{L_{\nu}}(X, T)=\sum_{\mu \in Z^{p}} X^{<\mu+\nu>} \otimes T^{(\mu)}
$$

for $X \in \mathscr{T}_{0}^{1}(M)$ and $T \in \mathscr{T}_{q}^{0}(M)$. It is clear that $\tilde{L}$ is a bilinear map over $R$. We now assert that the following

$$
\widetilde{L_{\nu}}(f X, T)=\tilde{L}_{\nu}(X, f T)
$$

holds for every $X \in \mathscr{T}_{0}^{1}(M), T \in \mathscr{T}_{q}^{0}(M)$ and $f \in \mathscr{T}_{0}^{0}(M)$. For, making use of Remark 2.7 and Lemma 3.1, we calculate as follows:

$$
\begin{aligned}
& \tilde{L}_{\nu}(f X, T)=\sum_{\mu}(f X)^{\langle\mu+\nu\rangle} \otimes T^{(\mu)} \\
& =\sum_{\mu} \sum_{\lambda} f^{(\lambda)} X^{<\lambda+\mu+\nu>} \otimes T^{(\mu)} \\
& =\sum_{\mu} \sum_{\lambda^{\prime}} f^{\left(\lambda^{\prime}-\mu-\nu\right)} X^{<\lambda>>} \otimes T^{(\mu)} \\
& =\sum_{\lambda^{\prime}} \sum_{\mu} X^{\left.<\lambda^{\prime}\right\rangle} \otimes f^{\left(\lambda^{\prime}-\mu-\nu\right)} T^{(\mu)}
\end{aligned}
$$




$$
\begin{aligned}
& =\sum_{\lambda^{\prime}} X^{\left.<\lambda^{\prime}\right\rangle} \otimes(f T)^{\left(\lambda^{\prime}-\nu\right)} \\
& =\sum_{\lambda} X^{<\lambda+\nu>} \otimes(f T)^{(\lambda)}=\tilde{L}_{\nu}(X, f T),
\end{aligned}
$$

which proves our assertion. Thus, we obtain a linear map $L_{\nu}$ of $\mathscr{T}_{q}^{1}(M)$ into $\stackrel{r, p}{\mathscr{T}}_{q}^{1}(T M)$ such that

$$
L_{\nu}(X \otimes T)=\sum_{\mu \in Z^{p}} X^{<\mu+\nu\rangle} \otimes T^{(\mu)}
$$

for $X \in \mathscr{T}_{0}^{1}(M)$ and $T \in \mathscr{T}_{q}^{0}(M)$. Put $\tilde{K}=L_{\nu}(K)$. It is now sufficient to prove (3.3) for $K=X \otimes T$ with $X \in \mathscr{T}_{q}^{0}(M)$ and $T \in \mathscr{T}_{q}^{0}(M)$. Using Corollary 3.4 and Lemma 2.6 , we can calculate as follows:

$$
\begin{aligned}
& \tilde{K}\left(X_{1}^{\left.<\lambda_{1}\right\rangle}, \cdots, X_{q}^{\left.<\lambda_{q}\right\rangle}\right)=\sum_{\mu} T^{(\mu)}\left(X_{1}^{\left.<\lambda_{1}\right\rangle}, \cdots, X_{q}^{\left\langle\lambda_{q}\right\rangle}\right) X^{\langle\mu+\nu\rangle} \\
& =\sum_{\mu}\left(T\left(X_{1}, \cdots, X_{q}\right)\right)^{(\mu-\lambda)} X^{\langle\mu+\nu\rangle} \\
& =\sum_{\mu^{\prime}}\left(T\left(X_{1}, \cdots, X_{q}\right)\right)^{(\mu \prime)} X^{\langle\mu++\nu+\lambda\rangle} \\
& =\left(T\left(X_{1}, \cdots, X_{q}\right) \cdot X\right)^{\langle\nu+\lambda\rangle}=\left(K\left(X_{1}, \cdots, X_{q}\right)\right)^{\langle\nu+\lambda\rangle} .
\end{aligned}
$$

The uniqueness of $\widetilde{K}$ is clear, since (3.3) holds for every $X_{i} \in \mathscr{T}_{0}^{1}(M)$ and $\lambda_{i} \in N(p, r)$.

Q.E.D.

Definition 3.6. For $K \in \mathscr{T}_{q}^{1}(M)$ and $\nu \in N(p, r)$, we denote $\tilde{K}$ in Lemma 3.5 by $\tilde{K}=K^{(\nu)}$ and call it the (v)-lift of $K$, i.e.

$$
K^{(\nu)}\left(X_{1}^{\left.<\lambda_{1}\right\rangle}, \cdots, X_{q}^{\left.<\lambda_{q}\right\rangle}\right)=\left(K\left(X_{1}, \cdots, X_{q}\right)\right)^{<\lambda+\nu>}
$$

for $X_{i} \in \mathscr{T}_{0}^{1}(M), \lambda_{i} \in N(p, r)$, where $\lambda=\sum \lambda_{i}$. We call $K^{(0)}$ the complete lift of $K$ to $\stackrel{r, p}{T} M$.

Lemma 3.7. For $K \in \mathscr{T}_{q}^{1}(M)(q \geqq 1)$ and $X \in \mathscr{T}_{0}^{1}(M)$, we have

$$
\alpha_{X}^{k}<\lambda>K^{(\mu)}=\left(\alpha_{X}^{k} K\right)^{(\mu+\lambda)}
$$

for $k \leq q$ and $\lambda, \mu \in N(p, r)$.

Proof. It suffices to prove (3.7) for $K=Y \otimes T$ with $Y \in \mathscr{T}_{0}^{1}(M), T \in \mathscr{T}_{q}^{0}(M)$. Using Lemma 3.3, we calculate as follows:

$$
\begin{aligned}
& \alpha_{X}^{k}<\lambda>K^{(\mu)}=\alpha_{X}^{k}<\lambda>\sum_{\nu} Y^{<\nu+\mu>} \otimes T^{(\nu)} \\
& =\sum_{\nu} Y^{\langle\nu+\mu>} \otimes \alpha_{X}^{k} k<>T^{(\nu)}
\end{aligned}
$$




$$
\begin{aligned}
& =\sum_{\nu} Y^{\langle\nu+\mu>} \otimes\left(\alpha_{X}^{k} T\right)^{(\nu-\lambda)} \\
& =\sum Y^{<\nu /+\lambda+\mu>} \otimes\left(\alpha_{X}^{k} T\right)^{\left(\nu^{\prime}\right)} \\
& =\left(Y \otimes \alpha_{X}^{k} T\right)^{(\lambda+\mu)}=\left(\alpha_{X}^{k} K\right)^{(\lambda+\mu)}
\end{aligned}
$$

Corollary 3.8. We have

$$
\alpha_{X}^{k}<0>K^{(\mu)}=\left(\alpha_{X}^{k} K\right)^{(\mu)}
$$

for every $X \in \mathscr{T}_{0}^{1}(M), K \in \mathscr{T}_{q}^{1}(M)$ and $\mu \in N(p, r)$.

\section{§4. Prolongations of almost complex structures.}

Lemma 4.1. For any $A, B \in \mathscr{T}_{1}^{1}(M)$, we have

$$
(A \circ B)^{(0)}=A^{(0)} \circ B^{(0)} \text {. }
$$

Let $I_{M} \in \mathscr{T}_{1}^{1}(M)$ be the $(1,1)$-tensor field of identity transformations of tangent spaces to $M$. Then, we have

$$
\left\langle I_{M}\right)^{(0)}=I_{\substack{p \cdot r \\ T M}}
$$

Proof. Making use of (3.6), we have, for any $X \in \mathscr{T}_{0}^{1}(M)$,

$$
\begin{aligned}
& A^{(0)} \circ B^{(0)}\left(X^{<\lambda>}\right)=A^{(0)}\left(B^{(0)}\left(X^{<\lambda>}\right)\right) \\
& =A^{(0)}\left((B(X))^{<\lambda>}\right)=(A B X)^{<\lambda>} \\
& =((A \circ B) X)^{<\lambda>}=(A \circ B)^{(0)}\left(X^{<\lambda>}\right)
\end{aligned}
$$

for every $\lambda \in N(p, r)$. Therefore we get (4.1).

To prove (4.2), let $I_{M}=\sum\left(\partial / \partial x_{i}\right) \otimes d x_{i}$ be the local expression of $I_{M}$, where $\left\{x_{i}, \cdots, x_{n}\right\}$ is a local coordinate system. Then, we have

$$
\begin{aligned}
\left(I_{M}\right)^{(0)} & =\sum_{i, \mu}\left(\frac{\partial}{\partial x_{i}}\right)^{\langle\mu\rangle} \otimes\left(d x_{i}\right)^{(\mu)} \\
& =\sum_{i, \mu} \frac{\partial}{\partial x_{i}^{(\mu)}} \otimes d x_{i}^{(\mu)}=I_{p, r},
\end{aligned}
$$

which proves (4.2).

Corollary 4.2. For any polynomial $P(x)$ of one variable $x$ with real coeffcients and for any $A \in \mathscr{T}_{1}^{1}(M)$, we have

$$
(P(A))^{(0)}=P\left(A^{(0)}\right)
$$


Proof. Use (4.1) and (4.2) repeatedly.

Q.E.D.

TheOREM 4.3. Let $J$ be an almost complex structure on $M$ with its Nijenhuis tensor $N_{J}$. Then, the bundle $\stackrel{r, p}{T} M$ of $p^{r}$-velocities in $M$ has an almost complex structure $J^{(0)}$ with its Nijenhuis tensor $\left(N_{J}\right)^{(0)}$.

Theorem 4.4. If a manifold $M$ is a complex manifold with almost complex structure $J$, so is the bundle $\stackrel{r, p}{T} M$ of $p^{r}$-velocities in $M$ with almost complex structure $J^{(0)}$.

\section{§5. Lifting of affine connections.}

Let $\nabla$ be the covariant differentiation defined by an affine connection of $M$.

THEOREM 5.1. There exists one and only one affine connection of $\stackrel{r, p}{T} M$ whose covariant differentiation $\tilde{\nabla}$ satisfies the following condition:

$$
\tilde{\nabla}_{X^{<\lambda>}} Y^{<\mu>}=\left(\nabla_{X} Y\right)^{<\lambda+\mu>}
$$

for every $X, Y \in \mathscr{T}_{0}^{1}(M)$ and $\lambda, \mu \in N(p, r)$.

Proof. Take a coordinate neighborhood $U$ with coordinate system $\left\{x_{1}, \cdots, x_{n}\right\}$ and let $\Gamma_{i j}^{k}$ be the connection components of $\nabla$ with respect to $\left\{x_{1}, \cdots, x_{n}\right\}$, i.e.

$$
\nabla_{\frac{\partial}{\partial x_{i}}} \frac{\partial}{\partial x_{i}}=\sum_{k} \Gamma_{i j}^{k} \frac{\partial}{\partial x_{k}}
$$

for $i, j=1, \cdots, n$. Let $\Gamma_{i j}^{\prime k}$ be the connection components of $\nabla$ with respect to another coordinate system $\left\{y_{1}, \cdots, y_{n}\right\}$ on $U$. Then, we have the following equalities:

$$
\Gamma_{i j}^{\prime k}=\sum_{a, b, c} \frac{\partial x_{b}}{\partial y_{i}} \frac{\partial x_{c}}{\partial x_{j}} \frac{\partial y_{k}}{\partial x_{a}} \Gamma_{b c}^{a}+\sum \frac{\partial^{2} x_{a}}{\partial y_{i} \partial y_{j}} \frac{\partial y_{k}}{\partial x_{a}}
$$

for $i, j, k=1,2, \cdots, n$. (cf. for instance [3] p. 27). Let $\left\{x_{i}^{(\nu)} \mid i=1, \cdots, n\right.$; $\nu \in N(p, r)\} \quad$ (resp. $\left.\left\{y_{i}^{(\nu)}\right\}\right)$ be the induced coordinate system on $(\pi)^{-1}(U)$. Define

$$
\tilde{\Gamma}_{(i, \nu)(j, \mu)}^{(k, \lambda)}=\left(\Gamma_{i j}^{k}\right)^{(\lambda-\nu-\mu)}
$$

for $i, j, k=1,2, \cdots, n ; \lambda, \mu, \nu \in N(p, r)$. We can now prove that there exists a connection $\tilde{\nabla}$ whose connection components with respect to $\left\{x_{i}^{(\nu)}\right\}$ are given 
by (5.4). For, we can verify (5.5) [6] for $\lambda, \mu, \nu \in N(p, r)$ in the same way as the proof of (5.5) [6], since we can use the equalities

$$
\frac{\partial f^{(\lambda)}}{\partial x_{i}^{(\mu)}}=\left(\frac{\partial f}{\partial x_{i}}\right)^{(\lambda-\mu)}
$$

for every $\lambda, \mu \in N(p, r)$ and $f \in C^{\infty}(U)$ (cf. Cor. 2.3).

Next, we shall verify the following

$$
\tilde{\nabla}_{X_{i}^{<\lambda>}} X_{j}^{<\mu>}=\left(\nabla_{X_{i}} X_{j}\right)^{<\lambda+\mu>}
$$

for every $i, j=1, \cdots n$ and $\lambda, \mu \in N(p, r)$, where we have put $X_{i}=\frac{\partial}{\partial x_{i}}$. Making use of Lemma 2.6 we calculate as follows:

$$
\begin{aligned}
& \tilde{\nabla}_{X_{i}^{\langle\lambda\rangle}} X_{j}^{\langle\mu\rangle}=\tilde{\nabla} \frac{\partial}{\partial x_{i}^{\langle\lambda\rangle}}\left(\frac{\partial}{\partial x_{j}^{(\mu)}}\right)=\sum_{\nu, k} \tilde{\Gamma}_{(i, \lambda),(j, \mu)}^{(\lambda, \nu)} \frac{\partial}{\partial x_{k}^{(\nu)}} \\
= & \sum_{\nu, k}\left(\Gamma_{i j}^{k}\right)^{(\nu-\lambda-\mu)} \frac{\partial}{\partial x_{k}^{(\nu)}}=\sum_{\nu, k}\left(\Gamma_{i j}^{k}\right)^{(\nu /)}\left(\frac{\partial}{\varrho x_{k}}\right)^{\langle\lambda+\mu+\nu\rangle} \\
= & \left(\sum_{k} \Gamma_{i j}^{k} \frac{\partial}{\partial x_{k}}\right)^{\langle\lambda+\mu\rangle}=\left(\nabla_{X_{i}} X_{j}\right)^{\langle\lambda+\mu\rangle} .
\end{aligned}
$$

Now, we shall verify

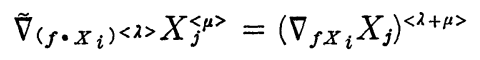

for $f \in C^{\infty}(U), i, j=1, \cdots, n$ and $\lambda, \mu \in N(p, r)$.

For, the left hand side of (5.6) is equal to

$$
\begin{aligned}
& \tilde{\nabla}_{\Sigma f(\nu)} X_{i}^{<\lambda+\nu>} X_{j}^{<\mu>}=\sum f^{(\nu)} \tilde{\nabla}_{X_{i}^{<\lambda+\nu>}} X_{j}^{<\mu>} \\
& =\sum f^{(\nu)}\left(\nabla_{X_{i}} X_{j}\right)^{\langle\lambda+\nu+\mu\rangle}=\left(f \cdot \nabla_{X_{i}} X_{j}\right)^{\langle\lambda+\mu\rangle}=\left(\nabla_{f X_{i}} X_{j}\right)^{\langle i+\mu\rangle} \text {, }
\end{aligned}
$$

which proves (5.6). Thus (5.1) is proved for $Y=\frac{\partial}{\partial x_{j}}$ and for every $X \in \mathscr{T}_{0}^{1}(M)$.

Finally, we shall verify (5.1) for $Y=\sum f_{i} X_{j} \in \mathscr{T}_{0}^{1}(M)$ as follows:

$$
\begin{aligned}
& \tilde{\nabla}_{X^{<\lambda>}}\left(\sum f_{i} X_{j}\right)^{<\mu>}=\tilde{\nabla}_{X^{<\lambda>}} \sum_{j, \nu} f_{j}^{(\nu)} X_{j}^{<\nu+\mu>} \\
& =\sum_{j, \nu}\left\{f_{j}^{(\nu)} \tilde{\nabla}_{X^{<\lambda>}} X_{j}^{\langle\nu+\mu>}+X^{\langle\lambda\rangle} f_{j}^{(\nu)} \cdot X_{j}^{\langle\nu+\mu>}\right\} \\
& =\sum_{j, \nu}\left\{f_{j}^{(\nu)}\left(\nabla_{X} X_{j}\right)^{\langle\nu+\lambda+\mu>}+\left(X f_{j}\right)^{(\nu-\lambda)} X_{j}^{\langle\nu+\mu>}\right\} \\
& =\sum_{j}\left\{\left(f_{j} \cdot \nabla_{X} X_{j}\right)^{\langle\lambda+\mu\rangle}+\left(X f_{j} \cdot X_{j}\right)^{\langle\lambda+\mu\rangle}\right\} \\
& =\left(\nabla_{X}\left(\sum f_{j} X_{j}\right)\right)^{<i+\mu>}
\end{aligned}
$$


The uniqueness of $\tilde{\nabla}$ is clear, since (5.1) holds for every $X, Y \in \mathscr{T}_{0}^{1}(M)$ and $\lambda, \mu \in N(p, r)$.

Q.E.D.

Definition 5.2. We denote $\tilde{\nabla}$ in Theorem 5.1 by $\tilde{\nabla}=\stackrel{r, p}{\nabla}$ and call it the complete lift of $\nabla$ to $\stackrel{r^{\prime}}{T} M$.

Proposition 5.3. Let $\tilde{T}, \tilde{R}$ be the torsion and the curvature tensor field of $\nabla=\stackrel{r, p}{\nabla}$. Then we have

$$
\widetilde{T}=T^{(0)} \text { and } R=\tilde{R},
$$

where $T^{(0)}$ and $R^{(0)}$ are the complete lift of $T$ and $R$ (cf. Def. 3.6).

Proof. Using the relation (3.6), we calculate as follows:

$$
\begin{aligned}
& T^{(0)}\left(X^{<\lambda>}, Y^{<\mu>}\right)=(T(X, Y))^{<\lambda+\mu>} \\
& =\left(\nabla_{X} Y-\nabla_{Y} X-[X, Y]\right)^{<\lambda+\mu>} \\
& =\tilde{\nabla}_{X^{<\lambda>}} Y^{<\mu>}-\tilde{\nabla}_{Y^{<\mu>}} X^{<\lambda>}-\left[X^{<\lambda>}, Y^{<\mu>}\right]=\tilde{T}\left(X^{<\lambda>}, Y^{<\mu>}\right)
\end{aligned}
$$

for every $X, Y \in \mathscr{T}_{0}^{1}(M)$ and $\lambda, \mu \in N(p, r)$, which proves $T^{(0)}=\tilde{T}$.

Similarly, we have:

$$
\begin{aligned}
& R^{(0)}\left(X^{<\lambda>}, Y^{<\mu>}\right) Z^{(\nu)}=(R(X, Y) Z)^{<\lambda+\mu+\nu\rangle} \\
& =\left(\nabla_{X} \nabla_{Y} Z-\nabla_{Y} \nabla_{X} Z-\nabla_{[X, Y]} Z\right)^{<\lambda+\mu+\nu>} \\
& =\tilde{\nabla}_{X}<\lambda>\tilde{\nabla}_{Y^{<\mu \nu}} Z^{<\nu>}-\tilde{\nabla}_{Y^{<\mu>}} \tilde{\nabla}_{X^{<\lambda>}} Z^{\langle\nu>}-\tilde{\nabla}_{\left[X^{<\lambda>}, Y^{<\mu>}\right]} Z^{\langle\nu>} \\
& =\tilde{R}\left(X^{<\lambda>}, Y^{<\mu>}\right) Z^{\langle\nu>}
\end{aligned}
$$

for every $X, Y, Z \in \mathscr{T}_{0}^{1}(M)$ and $\lambda, \mu, \nu \in N(p, r)$, which proves $R^{(0)}=\widetilde{R}$.

Q.E.D.

Proposition 5.4. For any $K \in \mathscr{T}_{q}^{s}(M)\left(s=0\right.$ or 1) and $X \in \mathscr{T}_{0}^{1}(M)$, we have

$$
\begin{aligned}
& \tilde{\nabla}_{X^{<0}}>K^{(\mu)}=\left(\nabla_{X} K\right)^{(\mu)}, \\
& \tilde{\nabla} K^{(\mu)}=(\nabla K)^{(\mu)}
\end{aligned}
$$

for every $\mu \in N(p, r)$.

Proof. It is sufficient to prove (5.8) for $K=Y \otimes T$, where $Y \in \mathscr{T}_{0}^{1}(M)$, $T \in \mathscr{T}_{q}^{0}(M)$. Now, since $K^{(\mu)}=\sum Y^{\langle v+\mu\rangle} \otimes T^{(\nu)}$, and since $\tilde{\nabla}_{X^{<0>}}$ is a derivation of $\stackrel{r, p}{\mathscr{T}}(T M)$, it suffices to verify $(5.8)$ in the special cases, where 
$K=f \in \mathscr{T}_{0}^{0}(M)$ and $K=Y \in \mathscr{T}_{0}^{1}(M)$ and $K=\theta \in \mathscr{T}_{1}^{0}(M)$.

If $K=f$, then we have

$$
\tilde{\nabla}_{X^{<0>}} f^{(\mu)}=X^{<0>} f^{(\mu)}=(X f)^{(\mu)}=\left(\nabla_{X} f\right)^{(\mu)} .
$$

If $K=Y$, then we have

$$
\tilde{\nabla}_{X^{<0}} Y^{<\mu>}=\left(\nabla_{X} Y\right)^{<\mu>}=\left(\nabla_{X} Y\right)^{(\mu)} .
$$

If $K=\theta$, then we have, for $\mu, \nu \in N(p, r)$ and $Y \in \mathscr{T}_{0}^{1}(M)$

$$
\begin{aligned}
& \left(\tilde{\nabla}_{X^{<0>}} \theta^{(\mu)}\right) Y^{\langle\nu\rangle}=\tilde{\nabla}_{X^{<0>}}\left(\theta^{(\mu)} Y^{\langle\nu\rangle}\right)-\theta^{(\mu)}\left(\tilde{\nabla}_{X^{<0>}} Y^{\langle\nu>}\right) \\
& \left.=\tilde{\nabla}_{X^{<0>}}(\theta(Y))^{(\mu-\nu)}-\theta^{(\mu)}\left(\left(\nabla_{X} Y\right)\right)^{\langle\nu>}\right) \\
& =\left(\nabla_{X} \theta(Y)\right)^{(\mu-\nu)}-\left(\theta\left(\nabla_{X} Y\right)\right)^{(\mu-\nu)} \\
& =\left(\left(\nabla_{X} \theta Y\right)^{(\mu-\nu)}=\left(\nabla_{X} \theta\right)^{(\mu)}\left(Y^{\langle\nu\rangle}\right),\right.
\end{aligned}
$$

and hence we get $\tilde{\nabla}_{X^{<0}} \theta^{(\mu)}=\left(\nabla_{X} \theta\right)^{(\mu)}$.

To prove (5.9), using Corollary 3.8, we calculate as follows

$$
\alpha_{X^{<0}} \tilde{\nabla} K^{(\mu)}=\tilde{\nabla}_{X^{<0}} K^{(\mu)}=\left(\nabla_{X} K\right)^{(\mu)}=\left(\alpha_{X}(\nabla K)\right)^{(\mu)}=\alpha_{X^{<0}}>(\nabla K)^{(\mu)} .
$$

Since $\left(X^{<0>}\right)_{[\varphi]_{r}}\left(X \in \mathscr{T}_{0}^{1}(M)\right)$ spans the tangent space to $\stackrel{r, p}{T M}$ at $[\varphi]_{r} \stackrel{r, p}{T} M$, we conclude that (5.9) holds.

Q.E.D.

Combining Proposition 5.3 and 5.4 we have proved the following

THEOREM 5.5. Let $T$ and $R$ be the torsion and the curavture tensor field of an affine connection $\nabla$ of $M$. According as $T=0, T=0, R=0$ or $\nabla R=0$, we have $T^{(0)}=0, \stackrel{r, p}{\nabla} T^{(0)}=0, R^{(0)}=0$ or $\stackrel{r, p}{\nabla} R^{(0)}=0$. In particular, if $M$ is affine locally symmetric with respect to $\nabla$, so is $\stackrel{r, p}{T} M$ with respect to $\stackrel{r, p}{\nabla}$.

\section{§6. Affine symmetric spaces.}

Let $\Phi: M \rightarrow N$ be a map of a manifold $M$ into another manifold $N$. Then, the map $\Phi$ induces a map $\stackrel{r, p}{T} \Phi$ of $\stackrel{r, p}{T} M$ into $\stackrel{r, p}{T} N$ as follows:

$$
(\stackrel{r, p}{T} \Phi)\left([\varphi]_{r}\right)=[\Phi \circ \varphi]_{r}
$$

for $[\varphi]_{r} \in \stackrel{r, p}{T} M$. The map $\stackrel{r, p}{T} \Phi$ is a well-defined differentiable map, which will be called the $(r, p)$-tangent to $\Phi$. It is clear that if $\Phi$ is a diffeomorphism then $\stackrel{r, p}{T \Phi}$ is also a diffeomorphism. 
Lemma 6.1. For any $f \in C^{\infty}(N)$, we have

$$
f^{(\mu)} \circ \stackrel{r, p}{T} \Phi=(f \circ \Phi)^{(\mu)}
$$

for every $\mu \in N(p, r)$.

Proof. Take a point $[\varphi]_{r} \stackrel{r, p}{T} M$. Then we have

$$
\begin{array}{ll}
\left(f^{(\mu)} \circ \stackrel{r, p}{T} \Phi\right)\left([\varphi]_{r}\right)=f^{(\mu)}\left([\Phi \circ \varphi]_{r}\right)=\frac{1}{\mu !}\left[\left(\frac{\partial}{\partial t}\right)^{\mu}(f \circ \mid(\Phi \circ \varphi))\right]_{t=0} \\
=\frac{1}{\mu !}\left[\left(\frac{\partial}{\partial t}\right)^{\mu}((f \circ \Phi) \circ \varphi)\right]_{t=0}=(f \circ \Phi)^{(\mu)}\left([\varphi]_{r}\right) . & \text { Q.E.D. }
\end{array}
$$

Lemma 6.2. Let $\Phi: M \rightarrow N$ be a diffeomorphism of $M$ onto $N$. Then for any $X \in \mathscr{T}_{0}^{1}(M)$ we have

$$
T^{r, p} \Phi\left(X^{<\lambda>}\right)=(T \Phi X)^{<\lambda>}
$$

for every $\lambda \in N(p, r)$.

Proof. Take a function $f \in C^{\infty}(N)$. Then, by making use of Lemma 6.1 and 2.1, we have, for any $\mu \in N(p, r)$ :

$$
\begin{aligned}
& T^{r, p} T \Phi\left(X^{<\lambda>}\right) f^{(\mu)}=X^{<\lambda>}\left(f^{(\mu)} \circ{ }^{r, p} T \Phi\right)=X^{<\lambda>}(f \circ \Phi)^{(\mu)} \\
& =(X(f \circ \Phi))^{(\mu-\lambda)}=((T \Phi X) f)^{(\mu-\lambda)}=(T \Phi X)^{<\lambda>} f^{(\mu)} .
\end{aligned}
$$

Since $f \in C^{\infty}(N)$ and $\mu \in N(p, r)$ are arbitrary, we get (6.3). Q.E.D.

Lemma 6.3. Let $\nabla$ (resp. $\left.\nabla^{\prime}\right)$ be an affine connection on $M($ resp. $N)$ and let $\Phi: M \rightarrow N$ be a diffeomorphism transforming $\nabla$ onto $\nabla^{\prime}$, i.e. we have

$$
T \Phi\left(\nabla_{X} Y\right)=\nabla_{T \Phi X}^{\prime} T \Phi Y
$$

for $X, Y \in \mathscr{T}_{0}^{1}(M)$. Then the map $\stackrel{r, p}{T} \Phi$ transforms $\stackrel{r, p}{\nabla}$ onto $\stackrel{r, p}{\nabla}$.

Proof. Put $\tilde{\Phi}=T \stackrel{r, p}{T} \Phi$. It suffices to verify

$$
\tilde{\Phi} \nabla_{X^{<\lambda>}} Y^{<\mu>}=\nabla_{\tilde{\Phi} X^{<\lambda>}} \tilde{\Phi} Y^{<\mu>}
$$

for every $X, Y \in \mathscr{T}_{0}^{1}(M)$ and $\lambda, \mu \in N(p, r)$. Now, by making use of Theorem 5.1 and Lemma 6.2, we see the left hand side of (6.7) is equal to 


$$
\begin{aligned}
& T^{r, p} \Phi\left(\nabla_{X} Y\right)^{\langle\lambda+\mu>}=\left(T \Phi\left(\nabla_{X} Y\right)\right)^{<\lambda+\mu>} \\
& =\left(\nabla_{T \Phi X}^{\prime} T \Phi Y\right)^{\langle\lambda+\mu>}=\stackrel{r, p}{\nabla}_{\left(T \Phi X^{<\lambda>}\right.}(T \Phi Y)^{\langle\mu>}=r_{\nabla^{\prime}, p}^{\prime} \tilde{\Phi} X^{<\lambda>} \tilde{\Phi} Y^{<\mu>} .
\end{aligned}
$$

Q.E.D.

Lemma 6.4. Take a point $x_{0} \in M$ and let $\Phi$ be a diffeomorphism of $M$ onto itself such that $\Phi\left(x_{0}\right)=x_{0}$ and that $T_{x_{0}} \Phi=-1_{T_{x_{0}} M}$. Consider the constant map $\gamma_{x_{0}}$ of $R^{p}$ into $M$ defined by $\gamma_{x_{0}}(u)=x_{0}$ for $u \in R^{p}$. Put $\tilde{x}_{0}=\left[\gamma_{x_{0}}\right]_{r}$. Then, we have $\stackrel{r, p}{T} \Phi)\left(\tilde{x}_{0}\right)=\tilde{x}_{0}$ and that

$$
\left.T_{\tilde{x}} T \Phi=-1_{T_{x_{0}}}{ }^{r} \dot{r}^{p} M\right)
$$

Proof. Take an element $\left.[\varphi]_{1} \in T_{\tilde{x}_{0}} \stackrel{r . d}{(T} M\right)$, where $\varphi: R \rightarrow \stackrel{r, p}{T} M$ with $\varphi(0)=\tilde{x}_{0}$. Making use of the same arguments as in the proof of Lemma 1.1 [5], we can find a differentiable map $\phi: R^{p+1} \rightarrow M$ such that $\varphi(t)=\left[\psi_{t}\right]_{r}$ for small $t$, where we have put $\phi_{t}(u)=\phi(t, u)$ for $t \in R$ and $u \in R^{p}$. Put $\phi^{u}(t)=\phi(t, u)$. Then, since $\varphi(0)=\left[\psi_{0}\right]_{r}=\tilde{x}_{0}=\left[\gamma_{x_{0}}\right]$, we can assume that $\phi(0, u)=x_{0}$ for small $u \in R^{p}$ (cf. the expression of $(\tilde{\psi})$ in the proof of Lemma 1.1 [5]). Take a coordinate neighborhood $U$ of $x_{0}$ with coordinate system $\left\{x_{1}, \cdots, x_{n}\right\}$. Put $x_{i, \nu}=x_{i}^{(\nu)}$ for $i=1, \cdots, n$ and $\nu \in N(p, r)$. Then $\left\{x_{i, \nu}\right\}$ is a coordinate system around $\tilde{x}_{0}$. We have to prove $T \stackrel{r, p}{T} \Phi\left([\varphi]_{1}\right)=-[\varphi]_{1}$, i.e. to prove $[\stackrel{r, p}{T} \Phi \circ \varphi]_{1}=$ $-[\varphi]_{1}$. To prove this, it suffices to prove the following

$$
\left(x_{i, \nu}\right)(1)\left([\stackrel{p \cdot p}{T} \Phi \circ \varphi]_{1}\right)=-\left(x_{i, \nu}\right)^{(1)}\left([\varphi]_{1}\right)
$$

for $i=1,2, \cdots, n$ and $\nu \in N(p, r)$.

Since $(\stackrel{r, p}{T} \Phi \circ \varphi)(t)=\stackrel{r, p}{T} \Phi(\varphi(t))=\stackrel{r, p}{T} \Phi\left(\left[\psi_{t}\right]_{r}\right)=\left[\Phi \circ \phi_{t}\right]_{r}$, we calculate as follows:

$$
\begin{aligned}
& \left(x_{i, \nu}\right)^{(1)}\left([\stackrel{r, p}{T} \Phi \circ \varphi]_{1}\right)=\left[\frac{\partial}{\partial t}\left(x_{i, \nu} \circ \stackrel{r, p}{T} \Phi \circ \varphi\right)\right]_{t=0}=\left[\frac{\partial}{\partial t}\left(x_{i, \nu}\left(\left[\Phi \circ \phi_{t}\right]_{r}\right)\right)\right]_{t=0} \\
& =\frac{1}{\nu !}\left[\frac { \partial } { \partial t } \left(\left[\left(\frac{\partial}{\partial u}\right)^{\nu}\left(x_{i} \circ \Phi \circ \phi_{t}\right)\right]_{t=0}\right.\right. \\
& =\frac{1}{\nu !}\left[\frac{\partial}{\partial t}\left(\left[\left(\frac{\partial}{\partial u}\right)^{\nu} x_{i}(\Phi(\psi(t, u)))\right]_{u=0}\right)\right]_{t=0} \\
& =\frac{1}{\nu !}\left[\left(\frac{\partial}{\partial u}\right)^{\nu}\left(\left[\frac{\partial}{\partial t} x_{i}\left(\Phi\left(\psi^{u}(t)\right)\right)\right]_{t=0}\right)\right]_{u=0}
\end{aligned}
$$

Now, making use of our assumption $T_{x_{0}} \Phi=-1_{T_{x_{0}} M}$ and the fact that $\phi^{u}(0)=$ 
$\phi(0, u)=x_{0}$ for small $u \in R^{p}$, we have

$$
\begin{aligned}
& {\left[\frac{\partial}{\partial t} x_{i}\left(\Phi\left(\psi^{u}(t)\right)\right)\right]_{t=0}=x_{i}^{(1)}\left(\left[\Phi \circ \psi^{u}\right]_{1}\right)=x_{i}^{(1)}\left(T \Phi\left[\psi^{u}\right]_{1}\right)} \\
& =-x_{i}^{(1)}\left(\left[\psi^{u}\right]_{1}\right)=-\left[\frac{\partial}{\partial t} x_{i}\left(\psi^{u}(t)\right)\right]_{t=0} \cdot
\end{aligned}
$$

Therefore, we can continue the above calculation as follows:

$$
\begin{aligned}
& \left(x_{i, \nu}\right)^{(1)}\left([\stackrel{r, p}{T} \Phi \circ \varphi]_{1}\right)=-\frac{1}{\nu !}\left[\left(\frac{\partial}{\partial u}\right)^{\nu}\left(\left[\frac{\partial}{\partial t} x_{i} \psi^{u}(t)\right]_{t=0}\right)\right]_{u=0} \\
& =-\frac{1}{\nu !}\left[\frac { \partial } { \partial t } \left(\left[\left(\frac{\partial}{\partial u}\right)^{\nu}\left(x_{i} \circ \psi_{t}\right)\right]_{u=0}=-\left(x_{i, \nu}\right)^{(1)}\left([\varphi]_{1}\right),\right.\right.
\end{aligned}
$$

which proves (6.6).

Q.E.D.

Corollary 6.5. Let $M$ be an affine symmetric space with affine connection $\nabla$. Let $\Phi: M \rightarrow M$ be the affine symmetry at a point $x_{0} \in M$. Then the $(r, p)$-tangent. $\stackrel{r, p}{T} \Phi$ to $\Phi$ is also the affine symmetry of $\stackrel{r, p}{T} M$ with affine connection $\stackrel{r, p}{\nabla}$ at the point $\tilde{x}_{0}$.

Proof. Since $\Phi$ leaves $\nabla$ invariant, $\stackrel{r, p}{T} \Phi$ also leaves $\stackrel{r, p}{\nabla}$ invariant by Lemma 6.3. Next, since $\Phi$ is an affine symmetry we see that $T_{x_{0}} \Phi=-1_{T x_{0} M}$ * Thus, by Lemma 6.4, we get (6.5), which means that $\stackrel{r, p}{T \Phi}$ is the affine symmetry at $\tilde{x}_{0}$. Q.E.D.

LemmA 6.6. Let $\nabla$ be an affine connection on a manifold $M$, and let $X \in \mathscr{T}_{0}^{1}(M)$ be an infinitesimal affine transofrmation of $\nabla$. Then, the $\langle\lambda\rangle$-lift $X^{<\lambda\rangle}$ of $X$ is also an infinitesimal affine transformation of $\tilde{\nabla}=\stackrel{r, p}{\nabla}$ on $\stackrel{r, p}{T} M$ for every $\lambda \in N(p, r)$.

Proof. A necessary and sufficient condition for $X$ to be an infinitesimal affine transformation of $M$ is that

$$
\mathscr{L}_{X} \circ \nabla_{X}-\nabla_{Y} \circ \mathscr{L}_{X}=\nabla_{[X, Y]}
$$

for every $Y \in \mathscr{T}_{0}^{1}(M)$; where $\mathscr{L}_{x}$ denotes the Lie derivation with respect to $X$. Therefore, we have to prove the following

$$
\mathscr{L}_{X<\lambda>} \circ \tilde{\nabla}_{\tilde{Y}} K-\nabla_{\tilde{Y}} \circ \mathscr{L}_{X<\lambda>} K=\tilde{\nabla}_{\left[X^{<\lambda>}, \tilde{Y}\right]} K
$$

for every $K \in \stackrel{r, p}{\mathscr{T}}(T M)$ and $\left.\tilde{Y} \in \mathscr{T}_{0}^{1} \stackrel{r, p}{(T)} M\right)$. To prove (6.7) it suffices to prove (6.7) for the special cases, where $\tilde{Y}=Y^{<\mu>}$ with $Y \in \mathscr{T}_{0}^{1}(M), \mu \in N(p, r)$ and $K=Z^{\langle\nu\rangle}$ or $\theta^{(\nu)}$ with $Z \in \mathscr{T}_{0}^{1}(M), \theta \in \mathscr{T}_{1}^{0}(M)$ and $\nu \in N(p, r)$. Moreover, to prove (6.7) for the case $K=\theta^{(\nu)}$ with $\theta \in \mathscr{T}_{1}^{0}(M)$, it suffices to prove (6.7) 
for $\theta=d f$ with $f \in \mathscr{T}_{0}^{0}(M)$.

If $K=Z^{<\nu\rangle}$, then we calculate as follows:

$$
\begin{aligned}
& \mathscr{L}_{X^{<\lambda>}} \tilde{\nabla}_{Y^{<\mu}} Z^{<\nu>}-\tilde{\nabla}_{Y^{<\mu>}} \mathscr{L}_{X}<\lambda>Z^{<\nu>} \\
& =\left[X^{\langle\lambda\rangle},\left(\nabla_{Y} Z\right)^{\langle\mu+\nu\rangle}\right]-\tilde{\nabla}_{Y^{<\mu>}}\left[X^{<\lambda\rangle}, Z^{\langle\nu\rangle}\right] \\
& =\left[X, \nabla_{Y} Z\right]^{\langle\lambda+\mu+\nu\rangle}-\left(\nabla_{Y}[X, Z]\right)^{<\lambda+\mu+\nu\rangle} \\
& \left.=\left(\left[X, \nabla_{Y} Z\right]-\nabla_{Y}[X, Z]\right)^{\langle\lambda+\mu+\nu\rangle}=\left(\mathscr{L}_{X} \circ \nabla_{Y}\right) Z-\left(\nabla_{Y} \circ \mathscr{L}_{X}\right) Z\right)^{<\lambda+\mu+\nu\rangle} \\
& =\left(\nabla_{[X, Y]} Z\right)^{\langle\lambda+\mu+\nu\rangle}=\nabla_{\left[X^{<\lambda>}, Y^{<\mu>}\right]} Z^{\langle\nu\rangle} \text {, }
\end{aligned}
$$

which proves (6.7) for $K=Z^{\langle\nu\rangle}$.

To prove (6.7) for the case $K=d f^{(\nu)}$ with $f \in \mathscr{T}_{0}^{n}(M)$, we first note that the following equalities hold:

$$
\left(\mathscr{L}_{X} \theta\right)(Y)=X(\theta(Y))-\theta([X, Y])
$$

$$
\left(\nabla_{X}(d f)\right)(Y)=X Y f-\left(\nabla_{X} Y\right) f
$$

for $X, Y \in \mathscr{T}_{0}^{1}(M), f \in \mathscr{T}_{0}^{0}(M)$ and $\theta \in \mathscr{T}_{1}^{0}(M)$.

Take a vector field $Z \in \mathscr{T}_{0}^{1}(M)$ and $\rho \in N(p, r)$. Making use of (6.8), (6.9), Lemma 2.5 and (5.1), we calculate as follows:

$$
\begin{aligned}
& \left.\left\{\mathscr{L}_{X^{<\lambda>}}\left(\tilde{\nabla}_{Y^{<\mu}}\left(d f^{(\nu)}\right)\right)-\tilde{\nabla}_{Y^{<\mu>}} \mathscr{L}_{X^{<\lambda>}}\left(d f^{(\nu)}\right)\right)\right\}\left(Z^{<\rho>}\right) \\
& \left.=X^{\langle\lambda\rangle}\left(\left(\tilde{\nabla}_{Y^{<\mu>}}\left(d f^{(\nu)}\right)\right)\left(Z^{\langle\rho\rangle}\right)\right)-\tilde{\nabla}_{Y^{<\mu>}} d f^{(\nu)}\right)\left(\left[X^{\langle\lambda\rangle}, Z^{\langle\rho\rangle}\right]\right)
\end{aligned}
$$

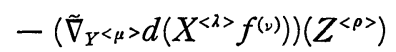

$$
\begin{aligned}
& =X^{<\lambda>}\left(Y^{<\mu>} Z^{<\rho>} f^{(\nu)}-\left(\tilde{\nabla}_{Y^{<\mu}} Z^{<\rho>}\right) f^{(\nu)}\right) \\
& -\left\{Y^{\langle\mu\rangle}\left[X^{\langle\lambda\rangle}, Z^{\langle\rho\rangle}\right] f^{(\nu)}-\left(\tilde{\nabla}_{Y^{<\mu>}}\left[X^{<\lambda\rangle}, Z^{\langle\rho\rangle}\right]\right) f^{(\nu)}\right\} \\
& -\left\{Y^{\langle\mu>} Z^{\langle\rho\rangle} X^{<\lambda>} f^{(\nu)}-\left(\tilde{\nabla}_{Y^{<\mu}} Z^{<\rho>}\right) X^{<\lambda>} f^{(\nu)}\right\} \\
& =\left[X\left\{Y Z f-\left(\nabla_{Y} Z\right) f\right\}-\left\{Y[X, Z] f-\left(\nabla_{Y}[X, Z] f-\left(\nabla_{Y}[X, Z]\right) f\right\}\right.\right. \\
& \left.-\left\{Y Z X f-\left(\nabla_{Y} Z\right) X f\right\}\right]^{(\nu-\mu-\rho-\lambda)} \\
& \left.=\left[\left\{\mathscr{L}_{X}\left(\nabla_{Y} d f\right)-\nabla_{Y} \mathscr{L}_{X} d f\right)\right\}(Z)\right]^{(\nu-\mu-\rho-\lambda)} \\
& =\left(\left(\nabla_{[X, Y]}(d f)(Z)\right)^{(\nu-\mu-\rho-\lambda)}=\left([X, Y] Z f-\left(\nabla_{[Y, Y]} Z\right) f\right)^{(\nu-\mu-\rho-\lambda)}\right. \\
& =\left[X^{<\lambda\rangle}, Y^{\langle\mu>}\right] Z^{\langle\rho\rangle} f^{(\nu)}-\left(\nabla_{\left[X^{<\lambda>}, Y^{<\mu>}\right.} Z^{\langle\rho>}\right) f^{(\nu)} \\
& =\left(\nabla_{\left[X^{<\lambda>}, Y^{<\mu>}\right]} d f^{(\nu)}\right)\left(Z^{<\rho>}\right) \text {, }
\end{aligned}
$$

which proves $(6.7)$ for $K=d f^{(\nu)}$, since $Z \in \mathscr{T}_{0}^{1}(M)$ and $\rho \in N(p, r)$ are arbitrary. Thus (6.7) holds for any $K$ and $\tilde{Y}$.

From Lemma 6.6 we obtain

Q.E.D. 
Proposition 6.7. If the group of affine transformations of $M$ with $\nabla$ is transitive on $M$, then the group of affine transformations of $\stackrel{r, p}{T} M$ with respect to $\stackrel{r, p}{\nabla}$ is transitive on $\stackrel{r, p}{T} M$.

From Proposition 6.7 and Corollary 6.5 we obtain the following

THEOREM 6.8. If $M$ is an affine symmetric space with connection $\nabla$, then $\stackrel{\boldsymbol{r}^{\mathrm{p}}}{\mathrm{T}} \mathrm{M}$ is also an affine symmetric space with connection $\stackrel{r, p}{\nabla}$.

\section{§ 7. Remarks.}

Let $P(M, \pi, G)$ be a principal fibre bundle with base $M$, projection $\pi$ and structure group $G$. We shall be able to prove that $\stackrel{r, p}{T} P(\stackrel{r, p}{T} M, \stackrel{r, p}{T} \pi, \stackrel{r, p}{T} G)$ becomes canonically a principal fibre bundle with structure group $\stackrel{\boldsymbol{r}_{i}}{T} G$, which is a Lie group by the natural group multiplication. Let $\omega$ be a connection form on $P$. Then by the same methods as in [5], we can construct the prolongation $\omega^{(r, p)}$ of $\omega$ to $\stackrel{r, p}{T P}$. If $P=F(M)$ is the frame bundle of $M$ then a linear connection on $M$ will induce a linear connection on $\stackrel{r, p}{T} M$ by the above procedure. We shall investigate the relationships between this procedure and the liftings of affine connections in $\$ 5$ in a forthcoming paper, where we shall also study the prolongations of $G$-structures to the tangent bundles of $p^{r}$-velocities, which will generalize the results in [4].

\section{REFERENCES}

[ 1 ] C. Ehresmann, Les prolongements d'une variété différentiable I. Calcul des jets, prolongement principal, C.R. Acad. Sci. Paris, 233(1951), 598-600.

[ 2 ] L Les prolongements d'une variété differentiable II, L'espace des jets d'ordre $r$ de $V_{n}$ dans $V_{m}$, ibid., 777-779.

[ 3 ] S. Helgason, Differential geometry and symmetric spaces, Acad. Press, 1962.

[4] A. Morimoto, Prolongations of G-structures to tangent bundles of higher order, Nagoya Math. J. 38(1970), 153-179.

[5] - Prolongations of connections to tangential fibre bundles of higher order, to appear.

[6 ] Liftings of tensor fields and connections to tangent bundles of higher order, to appear.

Nagoya University 